\title{
Bloqueadores neuromusculares en el síndrome de insuficiencia respiratoria progresiva aguda: metaanálisis
}

Neuromuscular blockers in acute respiratory distress syndrome: meta-analysis

Bloqueadores neuromusculares na síndrome de insuficiência respiratória progressiva aguda: metanálise

César Alejandro Rojas Gómez, * Alfredo Aisa Álvarez*

\section{RESUMEN}

Introducción: El uso de agentes bloqueadores neuromusculares (BNM) en adultos con síndrome de insuficiencia respiratoria progresiva aguda (SIRPA) se ha propuesto ampliamente. Sin embargo, la eficacia y seguridad de este tratamiento es incierta según la literatura más reciente. El objetivo de este estudio fue estimar la efectividad y la seguridad de los bloqueadores neuromusculares en el tratamiento del síndrome de insuficiencia respiratoria progresiva aguda.

Métodos: En esta revisión sistemática y de metaanálisis, se realizaron búsquedas en diferentes bases de datos y se compararon la administración de bloqueadores neuromusculares en comparación con placebo o no-tratamiento en pacientes con síndrome respiratoria progresiva aguda. Los títulos, resúmenes y textos completos de los artículos fueron seleccionados por duplicado por dos investigadores. Los datos para el diseño del estudio, las características de los pacientes, las intervenciones y los resultados se resumieron de forma independiente y por duplicado. Para obtener información adicional, se contactó a los autores de los estudios seleccionados por correo electrónico. Las guías GRADE se utilizaron para calificar la calidad de la evidencia. Calculamos las razones de riesgo (RR) y las razones de momios (OR) con intervalos de confianza de 95\% (IC de 95\%) para variables dicotómicas, mientras que para las variables continuas, obtuvimos la diferencia de medias y realizamos un metaanálisis de efectos aleatorios. El resultado primario fue la evaluación de mortalidad por cualquier causa, mortalidad en la Unidad de Cuidados Intensivos, la incidencia de efectos adversos y la evolución de los parámetros respiratorios.

Resultados: Se incluyeron seis estudios controlados aleatorizados (ECA). En comparación con el grupo de placebo o sin algún tratamiento, los bloqueadores neuromusculares se asociaron con una reducción significativa de la mortalidad por cualquier causa $(603$ [35.7\%] de 1,691 pacientes frente a 673 [40.5\%] de 1,660 pacientes; RR 0.84, [IC de 95\%: 0.74-0.95] p = 0.005, I2 33\%); así como disminución de la mortalidad en la UCI (RR 0.72 [IC de 95\%: 0.57-0.91]). En comparación con el grupo de placebo o sin algún tratamiento, el grupo de bloqueadores neuromusculares se asoció con una reducción significativa de los eventos adversos (RR 0.72 [IC de 95\%: 0.52 a 0.99], cuatro estudios controlados aleatorizados, 3,621 pacientes; $p=0.15,1264 \%$ ) y una significativa mejora en la relación $\mathrm{PaO}_{2} / \mathrm{FiO}_{2}(11.02 \mathrm{mmHg}$ [IC $95 \% 5.38$ a 16.66]; cuatro estudios controlados aleatorizados, 3,637 pacientes; $p=0.0001,1224 \%$ ).

Conclusiones: El uso de bloqueadores neuromusculares en adultos con síndrome de insuficiencia respiratoria progresiva aguda se asoció con una reducción significativa de la mortalidad por cualquier causa. Hubo menos eventos adversos y una mejora significativa en la relación con $\mathrm{PaO}_{2} / \mathrm{FiO}_{2}$ en el grupo de bloqueadores neuromusculares. Con base a nuestros resultados, recomendamos el uso de bloqueadores neuromusculares para pacientes con síndrome de insuficiencia respiratoria progresiva aguda de moderado a severo, en donde se necesite ventilación mecánica. Debido a la calidad moderada a baja de la evidencia, se requieren nuevos estudios aleatorizados con suficiente poder estadístico para confirmar estos hallazgos.

Palabras clave: Bloqueadores neuromusculares, síndrome de insuficiencia respiratoria progresiva aguda, metaanálisis, mortalidad.

\section{ABSTRACT}

Introduction: The use of neuromuscular blocking agents (NMBA) in adults with acute respiratory distress syndrome (ARDS) has been widely proposed. However, the efficacy and safety of this treatment is uncertain, according to the most recent literature. The objective of this study was to estimate the effect of neuromuscular blocking agents on acute respiratory distress syndrome.

\footnotetext{
* Centro Médico ABC. Ciudad de México.
}

Abreviaturas: $\mathrm{BNM}=$ agentes bloqueadores neuromusculares. $\mathrm{ECA}=$ estudio controlado aleatorizado. $\mathrm{OR}=$ razón de momios. $\mathrm{RR}=$ razón de riesgo. $\mathrm{UCl}=$ Unidad de Cuidados Intensivos. SIRPA = síndrome de insuficiencia respiratoria progresiva aguda.

Recepción: 18/09/2019. Aceptación: 02/12/2019.
Methods: In this systematic review and meta-analysis, different databases were searched, comparing the administration of neuromuscular blocking agents versus placebo or non-treatment in patients with acute respiratory distress syndrome. Titles, abstracts and full texts of the articles were selected in duplicate by two researchers. The data for the study design, patient characteristics, interventions and results were summarized independently and in duplicate. For additional information, the authors of the selected studies were contacted by email. The GRADE guides were used to rate the quality of the evidence. We calculate the risk ratios (RR) and odds ratios (OR) with $95 \%$ confidence intervals $(95 \% \mathrm{Cl})$ for dichotomous variables, while for the continuous variables we obtained the difference in means and performed a meta-analysis of random effects. The primary outcome was the evaluation of any-cause mortality, mortality in the Intensive Care Unit, the incidence of adverse effects and the evolution of respiratory parameters.

Results: Six randomized controlled studies (RCTs) were included. Compared to the placebo group or no treatment, neuromuscular blocking agents were associated with a significant reduction in any-cause mortality (603 [35.7\%] of 1,691 patients versus 673 [40.5\%] of 1,660 patients; RR 0.84 [Cl 95\%: 0.74-0.95] $p=0.005$ I2 33\%); as well as decreased mortality in the ICU (RR $0.72[95 \% \mathrm{Cl}$ $0.57-0.91]$ ). Compared to the placebo group or no treatment, the neuromuscular blocking agents group was associated with a significant reduction in adverse events (RR 0.72 [95\% Cl 0.52 to 0.99], four RCTs, 3,621 patients; $p=0.15 \mathrm{I} 2$ $64 \%$ ) and a significant improvement in the $\mathrm{PaO}_{2} / \mathrm{FiO}_{2}$ ratio $(11.02 \mathrm{mmHg}[95 \%$ Cl 5.38 to 16.66 ]; four $R C T s, 3,637$ patients; $p=0.0001$ I2 24\%).

Conclusions: The use of neuromuscular blocking agents in adults with acute respiratory distress syndrome was associated with a significant reduction in mortality from any cause. There were fewer adverse events and a significant improvement in the $\mathrm{PaO} / \mathrm{FiO}_{2}$ ratio in the neuromuscular blocking agents' group. Based on our results, we recommend the use of neuromuscular blocking agents for patients with moderate to severe ARDS who need mechanical ventilation. Due to the moderate to low quality of the evidence, new randomized studies with sufficient statistical power are required to confirm these findings. Keywords: Acute respiratory distress syndrome, neuromuscular blocking agents, metanalysis, mortality.

\section{RESUMO}

Introdução: $O$ uso de Agentes Bloqueadores Neuromusculares (BNM) em adultos com Síndrome da Insuficiência Respiratória Progressiva Aguda (SIRPA) tem sido amplamente proposto. No entanto, a eficácia e segurança deste tratamento são incertas, de acordo com a literatura mais recente. O objetivo deste estudo foi estimar a eficácia e a segurança do BNM no tratamento do SIRPA. Métodos: Nesta revisão sistemática e de metanálise, foram pesquisados diferentes bancos de dados, comparando a administração de BNM versus placebo ou não tratamento em pacientes com SIRPA. Os títulos, resumos e textos completos dos artigos foram selecionados em duplicado por dois pesquisadores. Os dados para o desenho do estudo, características do paciente, intervenções e resultados foram resumidos de forma independente e em duplicata. Para informações adicionais, os autores dos estudos selecionados foram contatados por email. Os guias GRADE foram usados para avaliar a qualidade da evidência. Calculamos as razões de risco (RR) e odds ratio (OR) com intervalos de confiança de 95\% (IC95\%) para variáveis dicotômicas, enquanto que para as variáveis contínuas obtivemos a diferença de médias e realizamos uma meta-análise de efeitos aleatórios. $O$ desfecho primário foi a avaliação da mortalidade por qualquer causa, mortalidade na Unidade de Terapia Intensiva, incidência de efeitos adversos e evolução dos parâmetros respiratórios.

Resultados: Foram incluídos seis estudos randomizados controlados (ERC). Comparado ao grupo placebo ou nenhum tratamento, os BNM foram associados a uma redução significativa na mortalidade por qualquer causa (603 [35.7\%] de 1691 pacientes versus 673 [40.5\%] de 1,660 pacientes; RR 0.84, [IC 95\%: 0.74 0.95 ] $p=0.005$ I2 33\%); bem como diminuição da mortalidade na UTI (RR 0.72; [IC95\% 0.57-0.91]). Comparado ao grupo placebo ou nenhum tratamento, o grupo BNM foi associado a uma reducão significativa de eventos adversos (RR 0.72, [IC 95\% 0.52 a 0.99], quatro ECR, 3,621 pacientes; $p=0.15 I 264 \%$ ) e uma melhora significativa na relação $\mathrm{PaO}, \mathrm{FiO}_{2}(11.02 \mathrm{mmHg}$, [IC $95 \% 5.38$ a 16.66]; quatro $E C R, 3637$ pacientes; $p=0.00011224 \%$ ). 
Conclusões: O uso de BNM em adultos com SIRPA foi associado a uma redução significativa na mortalidade por qualquer causa. Houve menos eventos adversos e uma melhora significativa na relação $\mathrm{PaO}_{2} / \mathrm{FiO}_{2}$ no grupo BNM. Com base em nossos resultados, recomendamos o uso de BNM para pacientes Com SIRPA moderada a grave que precisam de ventilação mecânica. Devido à qualidade moderada a baixa da evidência, novos estudos randomizados com poder estatístico suficiente são necessários para confirmar esses achados.

Palavras-chave: Bloqueadores neuromusculares, síndrome da insuficiência respiratória progressiva aguda, metanálise, mortalidade.

\section{INTRODUCCIÓN}

El síndrome de insuficiencia respiratoria progresiva aguda (SIRPA) es una enfermedad potencialmente mortal, que se caracteriza por una lesión pulmonar inflamatoria y que clínicamente se manifiesta por hipoxia y disminución de la distensibilidad pulmonar. ${ }^{1}$

EI SIRPA se puede desarrollar en el contexto de neumonía (35-50\% de los casos), en sepsis de origen no pulmonar (30\%), en aspiración de contenido gástrico $(10 \%)$ y en trauma $(10 \%){ }^{2}$ Varios otros escenarios menos comunes están asociados con el desarrollo de SIRPA, incluidos pancreatitis aguda, trasfusiones, inhalación de humo, entre otras causas. ${ }^{3}$

EI SIRPA fue descrito por primera vez en $1967,{ }^{4}$ y desde entonces distintos estudios han abordado los múltiples aspectos clínicos, tales como patogénesis, factores de riesgo y tratamiento. Sin embargo, a pesar de la intensa investigación, sólo se han descrito algunas terapias efectivas para el tratamiento del SIRPA. El abordaje terapéutico éste se basa en una estrategia multimodal que combina tanto estrategias no farmacológicas (ventilación basada en metas de protección alveolar o posición prono) como intervenciones farmacológicas, principalmente dirigidas al tratamiento de la causa subyacente. ${ }^{5}$

En la literatura se han reportado varias intervenciones farmacológicas para el abordaje del SIRPA, independientemente de su causa. De éstas, sólo el uso de bloqueadores neuromusculares (BNM) ha demostrado mejorar el pronóstico de los pacientes que presentan SIRPA. ${ }^{6}$ Sin embargo, literatura publicada más reciente ha cuestionado la utilidad de la terapia. En este sentido, según Moss y colaboradores, ${ }^{7}$ el uso de BNM no tiene beneficio en el tratamiento del SIRPA.

Teniendo en cuenta lo discordante de la evidencia, es necesario esclarecer el papel de los BNM en el tratamiento del SIRPA. Estos fármacos se utilizan con frecuencia en las Unidades de Terapia Intensiva, ${ }^{8}$ se ha reportado que entre $25 \%$ y $85 \%$ de los pacientes con SIRPA inscritos en distintos estudios recibieron BNM. ${ }^{9-12}$

La indicación exacta de los BNM en el tratamiento del SIRPA aún está por aclararse. Las primeras publicaciones sobre BNM fueron reportes de casos que obtuvieron mejoría en la relación $\mathrm{PaO}_{2} / \mathrm{FiO}_{2} \cdot{ }^{13-17}$ Hasta donde es de nuestro conocimiento, se han publicado tres metaanálisis sobre el tema. ${ }^{18-20}$ Sin embargo, en ninguno de los estudios previos se cita la evidencia más reciente.
Las propuestas sobre el mecanismo por el cual los BNM son beneficiosos en SIRPA son diversas: disminución en el biotrauma, ${ }^{21}$ en el consumo de oxígeno ${ }^{22-24}$ y en la presión transpulmonar. ${ }^{25-29}$

\section{MATERIAL Y MÉTODOS}

\section{Objetivos}

Evaluar la eficacia y seguridad del tratamiento con bloqueadores neuromusculares en pacientes críticos con síndrome de insuficiencia respiratoria progresiva aguda.

\section{Diseño del estudio}

Estudio tipo revisión sistemática y metaanálisis basado en estudios clínicos controlados y aleatorizados.

\section{Estudios incluidos}

Todos los estudios clínicos aleatorizados (randomized controlled clinical trials) que comparen la administración de bloqueadores neuromusculares en pacientes con síndrome de insuficiencia respiratoria progresiva aguda (según las definiciones más recientes ${ }^{30,31}$ ) contra los siguientes manejos: 1. «Placebo» y 2. «No-tratamiento».

Se definió como «no-tratamiento» al grupo control, en el cual se intenta que los elementos terapéuticos sean idénticos a los recibidos por el grupo experimental, exceptuando la administración del agente a ser investigado, en este caso los BNM.

No se restringió la búsqueda según el tipo, la duración o la dosis del BNM. Asimismo, fueron excluidos estudios en animales y estudios no controlados y aleatorizados. Excluimos también a niños, mujeres embarazadas y pacientes que no tuvieron diagnóstico de SIRPA.

No fue requerido el consentimiento informado o aprobación del consejo de ética para este estudio, dado que su realización se basó en estudios publicados previamente.

Estrategia de búsqueda y evaluación de la calidad de los artículos

Se realizaron búsquedas en las siguientes fuentes de cada base de datos hasta la fecha especificada (no existieron restricciones en el idioma de las publicaciones):

- Registro Cochrane Central de Estudios Controlados (CENTRAL) a través del Registro Cochrane de Estudios en Línea (CRSO) (hasta el 8 de junio de 2019).

- MEDLINE Ovid (Epub Ahead of Print, In-Process \& Other Non-Indexed Citations, Ovid MEDLINE (R) Daily y Ovid MEDLINE (R); desde 1946 hasta el 8 de junio de 2019). 
- Embase Ovid (desde 1974 hasta el 8 de junio de 2019).

- LILACS (base de datos de información sobre ciencias de la salud en América Latina y el Caribe; desde 1982 hasta el 8 de junio de 2019).

- ClinicalTrials.gov (www.clinicaltrials.gov) (hasta el 8 de junio de 2019).

- Plataforma de Registro Internacional de Estudios Clínicos (ICTRP) de la Organización Mundial de la Salud (www.who.int/trialsearch/) (hasta el 8 de junio de 2019).

Se utilizaron los siguientes términos clave en inglés para la realización de la búsqueda: "acute respiratory distress syndrome», "adult respiratory distress syndrome», "shock lung», "non-cardiogenic pulmonary edema», "ARDS», y «neuromuscular blocking agents», «neuromuscular blockade».

Se planeó identificar otros estudios potencialmente elegibles o publicaciones auxiliares mediante la búsqueda en las listas de referencias de los estudios incluidos, revisiones sistemáticas y metaanálisis. Además, se contactó con los autores de los estudios incluidos para identificar cualquier estudio adicional que se pudo haber pasado por alto. No se utilizaron resúmenes, ni resúmenes de congresos para la extracción de datos porque esta fuente de información no cumple con los requisitos de los estándares consolidados de reporte de estudios (CONSORT). ${ }^{32}$

Dos autores de la revisión (AA y CR) analizaron de forma independiente el título y/o el resumen de cada registro recuperado, para determinar qué estudios deberían evaluarse más a fondo. Se estudiaron a profundidad los artículos en su texto completo de todos los registros potencialmente relevantes, incluyendo materiales suplementarios y anexos. Resolvimos cualquier discrepancia por consenso o recurriendo a un tercer autor de la revisión (GC); si no se pudo resolver un desacuerdo, clasificamos el estudio como «estudios en espera de clasificación» y se estableció contacto con los autores de los estudios para obtener una aclaración. Presentamos un diagrama de flujo PRISMA adaptado para mostrar el proceso de selección de estudios (Figura 1).

Para la evolución de los estudios elegibles, los revisores (AA y CR) extrajeron los datos de forma independiente utilizando un formulario previamente acordado. Se resolvieron las discrepancias mediante discusión o, en caso necesario, consultamos al tercer autor de la revisión (GC). Ingresamos los datos en el software Review Manager (RevMan 2015). Cuando la información sobre cualquiera de los puntos anteriores no estaba clara, intentamos contactar a los autores de los estudios originales para que nos proporcionaran más detalles.
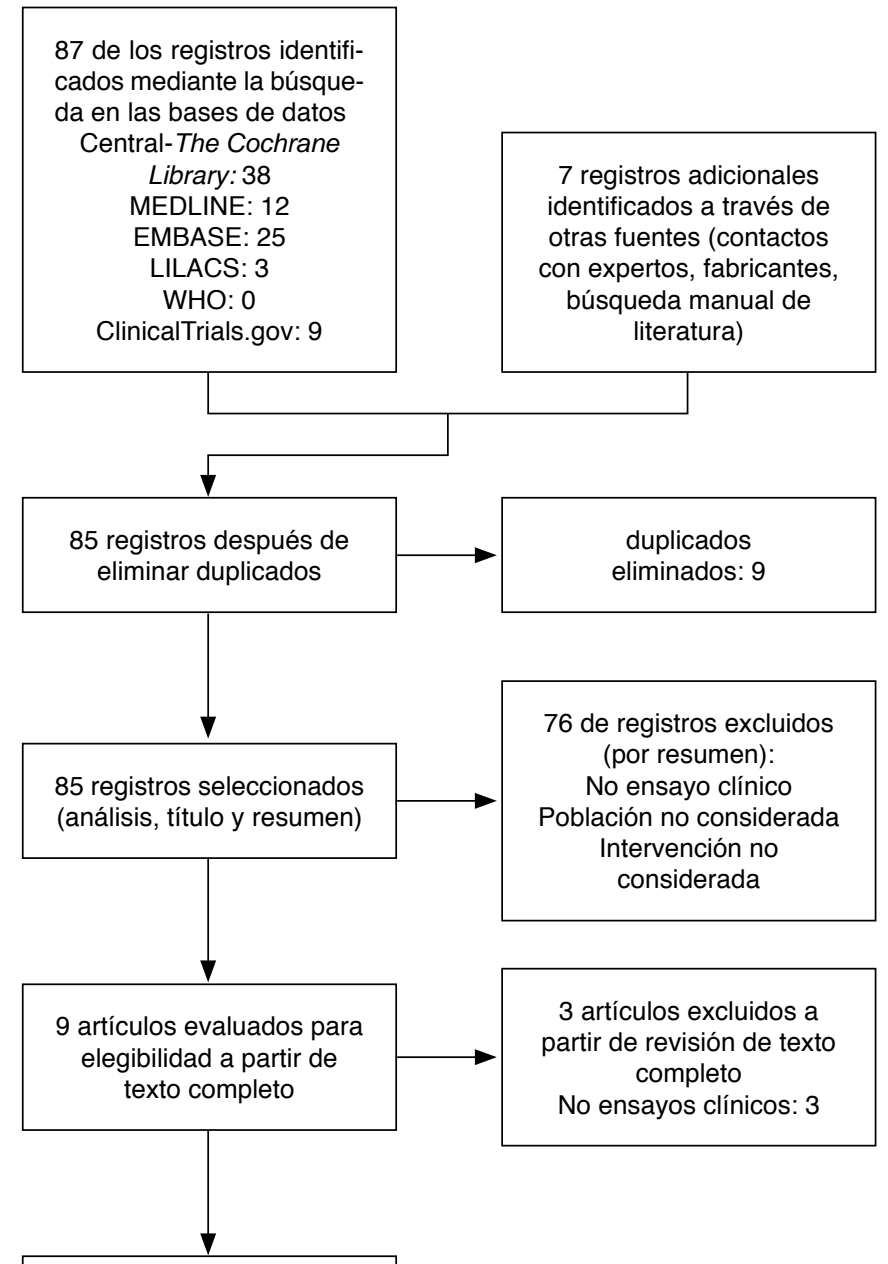

6 artículos considerados

para el análisis cualitativo

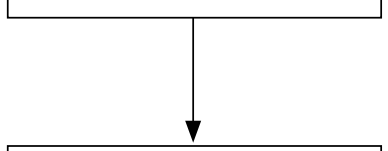

6 artículos considerados para el análisis

cuantitativo (metaanálisis)

\section{Figura 1: \\ Diagrama de flujo de la selección de estudios.}

De los estudios, se recolectaron los datos referentes a mortalidad, efectos adversos, grado de disfunción orgánica, parámetros ventilatorios y de oxigenación.

Los revisores evaluaron de forma independiente el «riesgo de sesgo» de cada estudio incluido. Resolvimos los desacuerdos por consenso o consultando a un tercer autor de la revisión (CG).

Asimismo, utilizamos la herramienta de evaluación Cochrane «Riesgo de sesgo 2», 33,34 asignando evaluaciones de riesgo de sesgo bajo, alto o poco claro. Evaluamos los elementos de sesgo individuales como se describe en el Manual Cochrane para Revisiones Sistemáticas de Intervenciones, de acuerdo con los criterios y las categorizaciones asociadas. 


\section{Análisis estadístico}

Se realizó el análisis estadístico mediante el software Review Manager (RevMan 2015). Se calculó el riesgo relativo (RR) con intervalos de confianza (IC) de 95\% para las variables dicotómicas. Se empleó la diferencia de medias (DM) para las variables continuas.

Evaluamos la heterogeneidad estadística en cada mediante el uso de las variables estadísticas $\mathrm{T}^{2}, \mathrm{I}^{2} \mathrm{y} \chi^{2}$, con un valor bajo de $p(<0.10)$ en la prueba de $\chi^{2}$ para heterogeneidad.

Se utilizó un metaanálisis de efectos fijos para combinar los datos cuando era razonable suponer que los estudios estaban estimando el mismo efecto subyacente del tratamiento; es decir, cuando los estudios están examinando la misma intervención, con poblaciones y métodos suficientemente similares. Si hubo heterogeneidad clínica suficiente para esperar que los efectos del tratamiento subyacente difirieran entre los estudios o si se detectó una heterogeneidad estadística sustancial, se utilizó un metaanálisis de efectos aleatorios. Cuando utilizamos análisis de efectos aleatorios, los resultados se presentan como el efecto promedio del tratamiento con intervalos de confianza de $95 \%$ y las estimaciones de $\mathrm{T}^{2}$ e $\mathrm{I}^{2}$.

\section{RESULTADOS}

Ochenta y siete estudios fueron identificados como potencialmente elegibles para su inclusión en esta revisión. Un total de 1,565 pacientes con SIRA participaron en los seis estudios finalmente incluidos, los cuales comparaban el bloqueo neuromuscular con placebo o ninguna intervención.

Un total de 698 participantes fueron asignados aleatoriamente al grupo de bloqueo neuromuscular. El grupo de comparación fue de 885 participantes: 162 en tratamiento con placebo y 723 en el grupo sin tratamiento. Sólo un estudio usó placebo como comparador. ${ }^{32}$

Respecto a la localización geográfica: una investigación se realizó en un centro en China, ${ }^{35}$ cuatro estudios se realizaron en múltiples centros en Francia ${ }^{32,36-38}$ y un estudio se realizó en 13 centros en EUA. ${ }^{7}$

Los estudios se realizaron de 2004 a 2018. La duración de las intervenciones varió de $48^{7,32,36-38}$ a 72 horas. ${ }^{35}$ La duración del seguimiento varió desde hasta el alta de la $\mathrm{UCI}^{36-38}$ hasta 12 meses. ${ }^{7}$ Ningún estudio finalizó antes de su fecha preespecificada.

La edad media de los participantes varió desde los 56 años en Moss 2019 hasta los 66 años en Guervilly 2017. Las características basales demográficas fueron similares entre los grupos de tratamiento en la mayoría de los estudios. La media de SAPS II varió de $44^{36}$ a 49 puntos. ${ }^{32}$ La puntuación SOFA varió de $9.5^{38}$ a 15.4 puntos. ${ }^{35}$ La lesión pulmonar directa fue el principal mecanismo de SIRPA en todos los estudios y la causa principal de la lesión pulmonar directa fue neumonía en la mayoría de los estudios (incluye neumonía por aspiración, adquirida en la comunidad, nosocomial y asociada con el ventilador). Sólo un estudio no informó la causa principal de lesión pulmonar directa. ${ }^{32}$

Los criterios de diagnóstico para SIRPA diferían entre los estudios. Tres trabajos utilizaron la definición de consenso estadounidense-europea. ${ }^{32,36,37}$ Tres trabajos utilizaron la definición de Berlín. 7,35,38 Un estudio utilizó Vecuronio ${ }^{35}$ mientras que el resto utilizaron cisatracurio.

\section{Evaluación de riesgo de sesgo}

Se utilizó la herramienta Cochrane para evaluación y resumen de riesgo de sesgos. Un estudio se clasificó como de bajo riesgo de sesgo y el resto de los estudios se clasificaron como alto riesgo o sesgo poco claro (Figura 2).

\section{Desenlaces clínicos}

\section{Mortalidad}

En comparación con el grupo con placebo o sin tratamiento, el bloqueo neuromuscular temprano se asoció con una reducción significativa de la mortalidad por cualquier causa (RR 0.84, IC de $95 \%$ : 0.74 a 0.95 , seis ECA [Tabla 1]) y en el riesgo de mortalidad en la UCI (RR 0.72; IC de 95\%: 0.57 a 0.91, cuatro ECA, 463 participantes).

El bloqueo neuromuscular se asoció con una reducción no significativa en la mortalidad de 21 a 28 días, así como de 60 a 90 días (Figura 3).

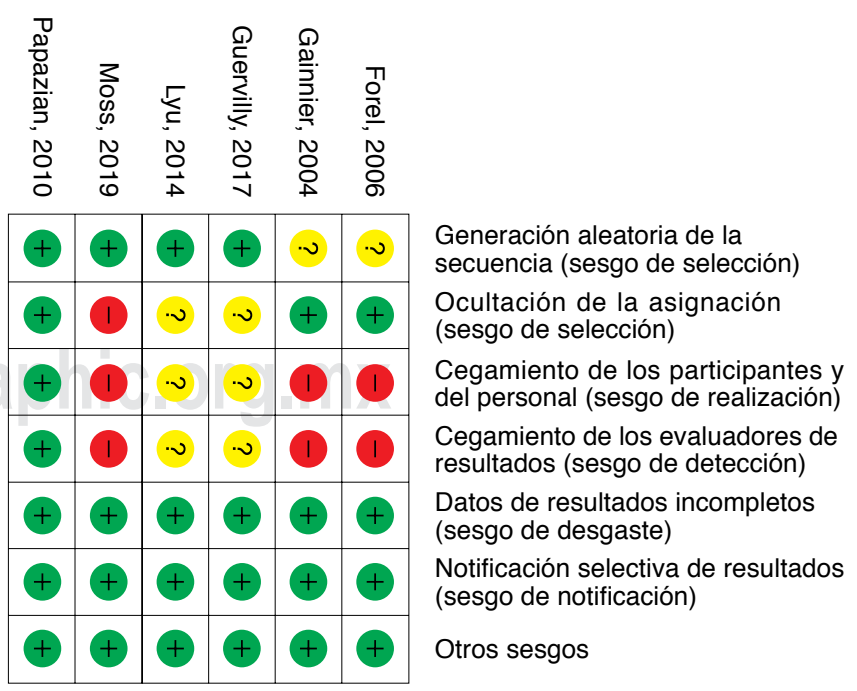

Figura 2: Resumen del riesgo de sesgo: juicios de los autores sobre cada elemento de riesgo de sesgo para cada uno de los estudios incluidos. 
Tabla 1: Resumen de la evidencia para desenlace, mortalidad por cualquier causa donde se evidencia una disminución en la mortalidad. Diferencias estadísticamente significativas.

\begin{tabular}{|c|c|c|c|c|c|c|c|c|c|c|c|c|}
\hline \multirow[b]{2}{*}{$\begin{array}{l}\text { Núm. de } \\
\text { estudios }\end{array}$} & \multirow[b]{2}{*}{$\begin{array}{l}\text { Diseño del } \\
\text { estudio }\end{array}$} & \multirow[b]{2}{*}{$\begin{array}{l}\text { Riesgo de } \\
\text { sesgo }\end{array}$} & \multirow[b]{2}{*}{$\begin{array}{l}\text { Inconsis- } \\
\text { tencia }\end{array}$} & \multirow[b]{2}{*}{$\begin{array}{c}\text { Evidencia } \\
\text { directa }\end{array}$} & \multirow[b]{2}{*}{ Imprecisión } & \multirow[b]{2}{*}{$\begin{array}{l}\text { Otras consi- } \\
\text { deraciones }\end{array}$} & \multicolumn{2}{|c|}{ Núm. de pacientes } & \multicolumn{2}{|c|}{ Efecto } & \multirow[b]{2}{*}{ Certeza } & \multirow[b]{2}{*}{ Importancia } \\
\hline & & & & & & & $\begin{array}{l}\text { Bloqueo } \\
\text { neuromus- } \\
\text { cular }\end{array}$ & $\begin{array}{l}\text { Placebo o } \\
\text { sin algún } \\
\text { tratamiento }\end{array}$ & $\begin{array}{l}\text { Relativo } \\
\text { (IC 65\%) }\end{array}$ & $\begin{array}{l}\text { Absoluto } \\
\text { (IC 65\%) }\end{array}$ & & \\
\hline \multicolumn{13}{|c|}{ Mortalidad por cualquier causa } \\
\hline 6 & $\begin{array}{l}\text { Ensayos } \\
\text { aleatoriza- } \\
\text { dos }\end{array}$ & No grave & Grave $^{a}$ & No grave & No grave & Ninguna ${ }^{b}$ & $\begin{array}{c}603 / 1,691 \\
(35.7 \%)\end{array}$ & $\begin{array}{c}673 / 1,660 \\
(40.5 \%)\end{array}$ & $\begin{array}{c}\text { RR } 0.84 \\
(0.74 \text { a } 0.95)\end{array}$ & $\begin{array}{c}65 \text { por } 1,000 \\
\text { (de } 105 \text { a } \\
20)\end{array}$ & Moderado & Crítico \\
\hline
\end{tabular}

a Encontramos heterogeneidad sustancial ( $I^{2}$ es mayor a $\left.30 \%\right)$.

${ }^{b}$ Sesgo de publicación detectado por funnel plot.

\section{Efectos adversos}

En comparación con el grupo con placebo o sin tratamiento, el bloqueo neuromuscular temprano se asoció con menos eventos de barotrauma en comparación con el grupo sin tratamiento o con placebo (RR 0.55; IC de 95\%: 0.35 a 0.85 , cuatro ECA) con diferencia significativa.

Hubo menos eventos de neumotórax en el bloqueo neuromuscular en comparación al grupo sin tratamiento o placebo (RR 0.46, IC de $95 \%$ : 0.28 a 0.77 , cuatro ECA) con diferencia significativa.

No hubo diferencias en la debilidad adquirida en la $\mathrm{UCl}^{34}$ por el alta entre los grupos (RR 1.06, IC de 95\%: 0.86 a 1.32 , cuatro ECA).

\section{Mejora de los parámetros respiratorios}

En comparación con el grupo con placebo o sin tratamiento, el bloqueo neuromuscular se asoció con una mejora significativa en $\mathrm{PaO}_{2} / \mathrm{FiO}_{2}(11.02 \mathrm{mmHg}$, IC de 95\%: 5.38 a 16.66, cuatro ECA). En comparación con el grupo con placebo o sin tratamiento, el bloqueo neuromuscular se asoció una mejora significativa en el grupo de bloqueo neuromuscular de $\mathrm{PaO}_{2} / \mathrm{FiO}_{2}$ a las 48 horas en comparación con placebo $(21.18 \mathrm{mmHg}$, IC de $95 \%$ : -0.17 a 42.53, $p=0,05$, tres $E C A)$.
En comparación con el grupo con placebo o sin tratamiento, el bloqueo neuromuscular se asoció con una reducción significativa en la PEEP $\left(-0.40 \mathrm{cmH}_{2} \mathrm{O}\right.$, IC de 95\%: -0.65 a -0.15 , cuatro ECA).

\section{DISCUSIÓN}

Nuestros resultados mostraron que el uso de BNM es beneficioso para pacientes con SIRPA con ventilación mecánica. El presente metaanálisis que incluyó seis estudios, demostró que el uso de BNM disminuye la mortalidad por cualquier causa y la mortalidad en la $\mathrm{UCl}$ en este grupo de pacientes. Además, los BNM mejoran la oxigenación y disminuyen la incidencia de barotrauma y neumotórax, sin aumentar la incidencia de debilidad adquirida en la terapia intensiva. Con base a nuestros resultados, recomendamos el uso de BNM para pacientes con SIRPA moderado a severo que necesitan ventilación mecánica.

\section{Investigación previa y mecanismos de acción}

La infusión de bloqueador neuromuscular de manera continua para el tratamiento del síndrome de insuficiencia respiratoria progresiva aguda se convirtió en una práctica común, después de que el estudio realizado por Papazian $(2010)^{32}$ demostrara reducción de la mortalidad. Distintos

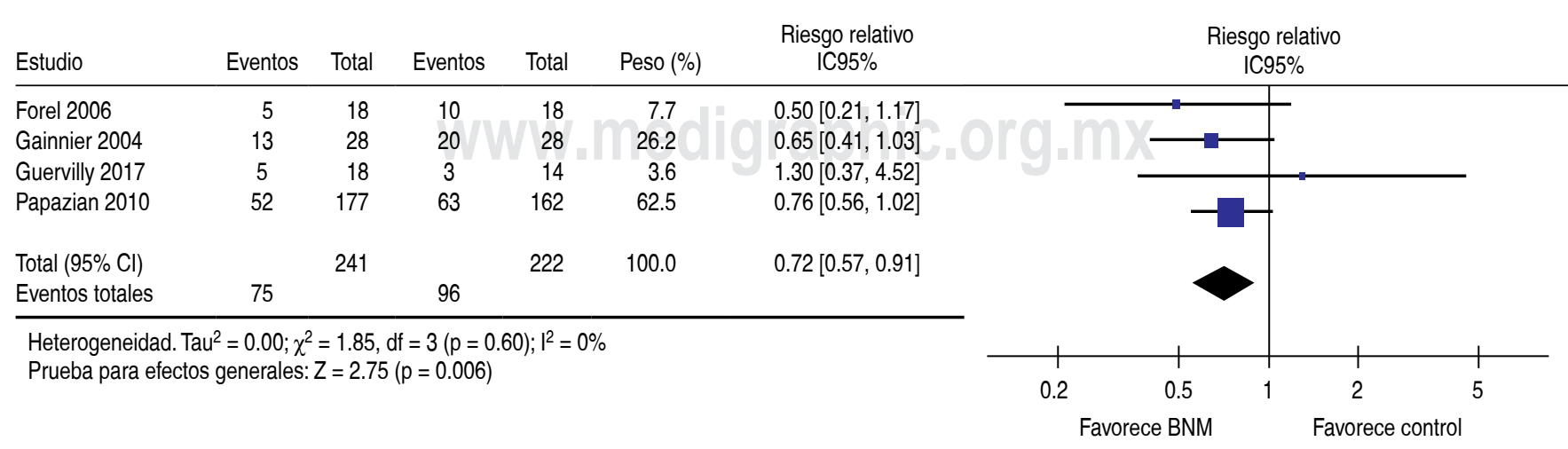

Figura 3: Análisis Forest Plot para desenlace mortalidad en la Unidad de Cuidados Intensivos donde se evidencia una disminución en la mortalidad. Diferencias estadísticamente significativas. 
trabajos realizados por el mismo grupo de investigadores encontraron resultados que apoyaban el uso de BNM. Sin embargo, el trabajo más reciente y más grande, Moss $(2019)^{7}$ no encontró ningún beneficio en su uso.

Aún existen dudas razonables sobre cuál debe ser el papel de los BNM, puesto que cada estudio tiene deficiencias metodológicas importantes que se deben considerar. Respecto al análisis estadístico, es importante señalar que el estudio de Papazian, los autores utilizaron la «mortalidad hospitalaria medida a los 90 días» como desenlace primario. Sin embargo, tanto el resumen como el resto del manuscrito reporta «mortalidad a 90 días» como resultado primario. La distinción es importante porque la «mortalidad a los 90 días» (es decir, evaluar si un paciente está vivo o muerto a los 90 días después de la aleatorización, independientemente de su ubicación) es una medida de resultado más sólida que la «mortalidad hospitalaria medida a los 90 días» (es decir, evaluar la mortalidad sólo durante la estadía en el hospital dentro de los primeros 90 días de la aleatorización), ya que este último no tiene en cuenta a los pacientes que podrían haber muerto después del alta hospitalaria dentro de los primeros 90 días. ${ }^{39}$

Del mismo modo, en el estudio de Papazian, ${ }^{32}$ las curvas de mortalidad comienzan a separarse después de aproximadamente 18 días después de la aleatorización. Aún no se ha podido entender del todo este lapso que tarda en presentarse los efectos benéficos de los agentes bloqueadores neuromusculares y las teorías que proponen su explicación resultan controversiales. Por ejemplo, se ha hecho hincapié en el potencial efecto antiinflamatorio directo de los BNM, aunque esto parece poco probable dada la falta de efecto sobre la mortalidad de agentes antiinflamatorios mucho más potentes en estudios previos. ${ }^{31}$ Por un lado, es poco probable que la mejoría en la oxigenación sea la principal explicación de los resultados positivos del estudio de Papazian, ya que las mediciones de intercambio de gases fueron en términos prácticos, esencialmente las mismas en el grupo control y experimental durante el periodo de administración del agente de bloqueo neuromuscular.

Por otro lado, el estudio de Moss se detuvo temprano por futilidad después de demostrar que era obvio que no había beneficio del bloqueo neuromuscular en los pacientes con SIRPA. Los autores sugieren que la evolución en las mejoras en la atención en terapia intensiva, en específico la reducción de la sedación, dieron como resultado un resultado negativo en relación con los estudios publicados anteriormente. Entonces se podría deducir que el bloqueo neuromuscular salva vidas en la misma intensidad en que la sedación profunda es letal: la sedación más ligera en el brazo de control debería haber salvado un número alto de vidas para borrar los beneficios de supervivencia de los BNM. Sin embargo, este complicado razonamiento será difícilmente la causa de las diferencias en mortalidad; de hecho, existe una diferencia fundamental entre ambos estudios: casi la mitad de los pacientes en los análisis franceses, incluido el de Papazian, fueron colocados en posición prono, mientras que sólo $16 \%$ de los pacientes en el estudio de Moss fueron pronados. Por razones que no están muy claras, los centros estadounidenses han tardado en adoptar la práctica de la posición prono. ${ }^{40,41}$ En este estudio de Moss, se dejó a discreción del médico tratante individual, y en cinco de seis pacientes, no utilizaron la opción.

Se desconoce si el bloqueo neuromuscular temprano es más efectivo aunado al posicionamiento en prono, pero es una posible explicación para los resultados tan diferentes de los estudios franceses y americanos. Resultaría útil un estudio que compare la utilidad de los BNM en un contexto de estandarización de la posición en prono.

\section{Fortalezas y limitaciones del estudio}

La fortaleza de nuestro metaanálisis es que se incorporaron datos de seis ECA. Es el metaanálisis más grande realizado hasta la fecha sobre este tema en el mundo. En la pirámide de la medicina basada en la evidencia, una revisión sistemática de ensayos controlados se encuentra en la parte superior. Hasta donde tenemos conocimiento, éste es el metaanálisis más grande en terapia intensiva realizado por un grupo de investigación mexicano.

La mayor limitación del estudio es el grado de sesgo de las publicaciones originales que impactan en el desarrollo de nuestro estudio.

\section{CONCLUSIONES}

Esta revisión sugiere que el bloqueo neuromuscular temprano produce una reducción en la mortalidad por cualquier causa y en la UCI en pacientes con SIRPA moderado a severo.

Del mismo modo, no encontramos un aumento en los efectos adversos en pacientes tratados con bloqueo neuromuscular, de hecho, se evidenció disminución en la incidencia de neumotórax en los pacientes tratados con bloqueadores neuromusculares, sin aumento en la debilidad adquirida en la terapia intensiva.

\section{BIBLIOGRAFÍA}

1. Rawal G, Yadav S, Kumar R. Acute respiratory distress syndrome: an update and review. J Trans/ Int Med. 2018;6(2):74-77.

2. Pham T, Rubenfeld GD. The epidemiology of acute respiratory distress syndrome. A 50th birthday review. Am J Respir Crit Care Med. 2017;195(7):860-870.

3. Matthay MA, Zemans RL, Zimmerman GA, Arabi YM, Beitler JR, Mercat A, et al. Acute respiratory distress syndrome. Nat Rev Dis Primers. 2019;5(1):18.

4. Ashbaugh D, Bigelow DB, Petty T, Levine B. Acute respiratory distress in adults. Lancet. 1967;290(7511):319-323. 
5. Baron RM, Levy BD. Recent advances in understanding and treating ARDS. F1000Res. 2016;5. pii: F1000 Faculty Rev-725.

6. Papazian L, Aubron C, Brochard L, Chiche JD, Combes A, Dreyfuss D, et al. Formal guidelines: management of acute respiratory distress syndrome. Ann Intensive Care. 2019;9(1):69.

7. National Heart, Lung, and Blood Institute PETAL Clinical Trials Network, Moss M, Huang DT, Brower RG, Ferguson ND, Ginde $A A$, et al. Early neuromuscular blockade in the acute respiratory distress syndrome. N Engl J Med. 2019;380(21):1997-2008.

8. Szakmany T, Woodhouse T. Use of cisatracurium in critical care: a review of the literature. Minerva Anestesiol. 2015;81:450-460.

9. Brower RG, Matthay MA, Morris A, Schoenfeld D, Thompson BT, Wheeler A, et al. Ventilation with lower tidal volumes as compared with traditional tidal volumes for acute lung injury and the acute respiratory distress syndrome. $N$ Engl $\mathrm{J}$ Med. 2000;342:1301-1308.

10. Brower RG, Lanken PN, Maclntyre N, Matthay MA, Morris A, Ancukiewicz M, et al. Higher versus lower positive end-expiratory pressures in patients with the acute respiratory distress syndrome. N Engl J Med. 2004;351:327-336.

11. Arroliga A, Frutos-Vivar F, Hall J, Esteban A, Apezteguia C, Soto $L$, et al. Use of sedatives and neuromuscular blockers in a cohort of patients receiving mechanical ventilation. Chest $J$. 2005;128:496-506.

12. Guerin C, Reignier J, Richard JC, Beuret P, Gacouin A, Boulain $\mathrm{T}$, et al. Prone positioning in severe acute respiratory distress syndrome. N Engl J Med. 2013;368:2159-2168.

13. Stark AR, Bascom R, Frantz ID. Muscle relaxation in mechanically ventilated infants. J Pediatr. 1979;94:439-443.

14. Bishop MJ. Hemodynamic and gas exchange effects of pancuronium bromide in sedated patients with respiratory failure. J Am Soc Anesthesiol. 1984;60:369-371.

15. Coggeshall JW, Marini JJ, Newman JH. Improved oxygenation after muscle relaxation in adult respiratory distress syndrome. Arch Intern Med. 1985;145:1718-1720.

16. Lagneau F, D'honneur G, Plaud B, Mantz J, Gillart T, Duvaldestin $\mathrm{P}$, et al. A comparison of two depths of prolonged neuromuscular blockade induced by cisatracurium in mechanically ventilated critically ill patients. Intensive Care Med. 2002;28:1735-1741.

17. Bourenne J, Hraiech S, Roch A, Gainnier M, Papazian L, \& Forel JM. Sedation and neuromuscular blocking agents in acute respiratory distress syndrome. Ann Transl Med. 2017;5(14).

18. Alhazzani W, Alshahrani M, Jaeschke R, Forel JM, Papazian $\mathrm{L}$, Sevransky J, et al. Neuromuscular blocking agents in acute respiratory distress syndrome: a systematic review and metaanalysis of randomized controlled trials. Crit Care. 2013;17:R43.

19. Neto AS, Pereira VGM, Esposito DC, Damasceno MCT, Schultz MJ. Neuromuscular blocking agents in patients with acute respiratory distress syndrome: a summary of the current evidence from three randomized controlled trials. Ann Intensive Care. 2012;2:33.

20. Tao W, Yang LQ, Gao J, Shao J. Neuromuscular blocking agents for adult patients with acute respiratory distress syndrome: A meta-analysis of randomized controlled trials. J Trauma Acute Care Surg. 2018;85(6):1102-1109.

21. Fanelli V, Morita Y, Cappello P, Ghazarian M, Sugumar $B$, Delsedime L, et al. Neuromuscular blocking agent cisatracurium attenuates lung injury by inhibition of nicotinic acetylcholine receptor-a1. J Am Soc Anesthesiol. 2016;124:132-140.

22. Marik PE, Kaufman D. The effects of neuromuscular paralysis on systemic and splanchnic oxygen utilization in mechanically ventilated patients. Chest. 1996;109:1038-1042.

23. Freebairn RC, Derrick J, Gomersall CD, Young RJ, Joynt GM. Oxygen delivery, oxygen consumption, and gastric intramucosal $\mathrm{pH}$ are not improved by a computer-controlled, closed- loop, vecuronium infusion in severe sepsis and septic shock. Crit Care Med. 1997;25:72-77.

24. Putensen C, Mutz NJ, Putensen-Himmer G, Zinserling J. Spontaneous breathing during ventilatory support improves ventilation-perfusion distributions in patients with acute respiratory distress syndrome. Am J Respir Crit Care Med. 1999;159:1241-1248.
25. Hraiech S, Yoshida T, Papazian L. Balancing neuromuscular blockade versus preserved muscle activity. Curr Opin Crit Care. 2015;21:26-33.

26. Slutsky AS. Neuromuscular blocking agents in ARDS. $N$ Engl J Med. 2010;363:1176-1180.

27. Bolaki M, Amargianitakis V, Georgopoulos D, Guervilly C, \& Papazian L. Effects of neuromuscular blockers on transpulmonary pressures in moderate to severe acute respiratory distress syndrome. Intensive Care Med. 2017;43(4):600.

28. Yoshida T, Uchiyama A, Matsuura N, Mashimo T, Fujino Y. The comparison of spontaneous breathing and muscle paralysis in two different severities of experimental lung injury. Crit Care Med. 2013;41:536-545.

29. Slutsky AS, Villar J. Early paralytic agents for ARDS? Yes, no, and Sometimes. N Engl J Med. 2019;380:2061-2063.

30. Schünemann HJ, Oxman AD, Vist GE, Higgins JPT, Deeks JJ, Glasziou P, et al. On behalf of the Cochrane Applicability and Recommendations Methods Group. Chapter 12: Interpreting results and drawing conclusions. In: Higgins JPT, Churchill R, Chandler J, Cumpston MS (editors). Cochrane Handbook for Systematic Reviews of Interventions Version 5.2.0 (updated June 2017). The Cochrane Collaboration, 2017. Available from: www.training.cochrane.org/handbook.

31. Raghavendran K, Pryhuber GS, Chess PR, Davidson BA, Knight PR, Notter RH. Pharmacotherapy of acute lung injury and acute respiratory distress syndrome. Curr Med Chem. 2008;15(19):1911-1924.

32. Papazian L, Forel JM, Gacouin A, Penot-Ragon C, Perrin G, Loundou A, et al. Neuromuscular blockers in early acute respiratory distress syndrome. N Engl J Med. 2010;363(12):1107-1116.

33. Force ADT, Ranieri VM, Rubenfeld GD. Acute respiratory distress syndrome. JAMA. 2012;307(23):2526-2533.

34. Paternostro-Sluga T, Grim-Stieger M, Posch M, Schuhfried O, Vacariu G, Mittermaier C, et al. Reliability and validity of the Medical Research Council (MRC) scale and a modified scale for testing muscle strength in patients with radial palsy. $J$ Rehabil Med. 2008;40(8):665-671.

35. Lyu G, Wang X, Jiang W, Cai T, Zhang Y. Clinical study of early use of neuromuscular blocking agents in patients with severe sepsis and acute respiratory distress syndrome. Zhonghua Wei Zhong Bing Ji Jiu Yi Xue. 2014;26(5):325-329.

36. Gainnier M, Roch A, Forel JM, Thirion X, Arnal JM, Donati S, et al. Effect of neuromuscular blocking agents on gas exchange in patients presenting with acute respiratory distress syndrome. Crit Care Med. 2004;32(1):113-119.

37. Forel JM, Roch A, Marin V, Michelet P, Demory D, Blache JL, et al. Neuromuscular blocking agents decrease inflammatory response in patients presenting with acute respiratory distress syndrome. Crit Care Med. 2006;34(11):2749-2757.

38. Guervilly C, Bisbal M, Forel JM, Mechati M, Lehingue S, Bourenne $\mathrm{J}$, et al. Effects of neuromuscular blockers on transpulmonary pressures in moderate to severe acute respiratory distress syndrome. Intensive Care Med. 2017;43(3):408-418.

39. Yegneswaran B, Murugan R. Neuromuscular blockers and ARDS: thou shalt not breathe, move, or die! Crit Care. 2011;15(5):311.

40. Lamm WJ, Graham MM, Albert RK. Mechanism by which the prone position improves oxygenation in acute lung injury. $A m \mathrm{~J}$ Respir Crit Care Med. 1994;150(1):184-193.

41. Fan E, Del Sorbo L, Goligher EC, Hodgson CL, Munshi L, Walkey AJ, et al. An Official American Thoracic Society/European Society of Intensive Care Medicine/Society of Critical Care Medicine Clinical Practice Guideline: Mechanical Ventilation in Adult Patients with Acute Respiratory Distress Syndrome. Am J Respir Crit Care Med. 2017;195(9):1253-1263.

\section{Correspondencia:}

Dr. Alfredo Aisa Álvarez

Av. Carlos Fernández Graef 154,

Santa Fe, Contadero, Cuajimalpa de Morelos,

05330 Ciudad de México, CDMX

Tel: 5585839499

E-mail: alfredoaisa@hotmail.com 